Historic, Archive Document

Do not assume content reflects current scientific knowledge, policies, or practices. 


\title{
WHOLESALE PRICE LIST OF GLADIOLUS
}

\author{
GROWN ON \\ HIM HTI, FARM \\ R. C. BELLARD \\ SUCCESSOR TO A. H. AUSTIN CO.
}

Prices per 100 unless otherwise noted. 25 at 100 rate. 1000 at 9 times 100 rate. 250 at 1000 rate. Orders for less than 25 will be charged at dozen prices.

PRICES F. O. B. WAYLAND, O.

\section{DISCOUNTS}

Orders of $\$ 25.00$ to $\$ 100.00$ from this list will be subject to $5 \%$ discount. On orders of $\$ 100.00$ or more $10 \%$ discount will be allowed. Our terms are cash where credit has not been established with us. We will hold bulbs for spring delivery on a deposit of $25 \%$.

R. C. BELLARD :-: WAYLAND, OHIO

Telephone and Telegraph-Ravenna, Ohio 


\section{STANDARD VARIETIES}

Albania-Pure silvery white.

Alice Tiplady-Large, orange color

America-Lavender pink

Anna Eberius-Purple

Beaconflame.-Flame scarlet

Bertrex-White, lilac markings in throat. Strong grower

Betty Joy-Soft creamy white, flushed LaFrance pink.

Many flowers open.

Break O'Day-Soft cream, flushed pink. Very delicate. Early

Byron L. Smith-Lavender pink, pale yellow throat. Very fine

Captain Boynton-Lavender with darker throat. Good

Carmen Sylva-Choice white. Tall. Slender.

Crimson Glow-Crimson. Light markings in throat. Tall

Crinkles-Peach blossom. Pink.

Dr. F. E. Bennett-“House-afire" red. Extra good.

E. J. Shaylor-Deep rose pink. Ruffled. Early.

Elf-Lemon yellow

Elizabeth Tabor-Similar to Pendleton. Much earlier

Empress of India-Very dark velvety red

Evelyn Kirtland-Dark rosy pink shading to shell pink. Tall.

Flora-Canary yellow. Large flowers. Tall.

Gertrude Errey-Shell pink, darker shading in throat

Gold Drop-Large yellow. Early

Gold Eagle-Deep yellow. Ruffled. Early.

Golden Dream-Clear, deep yellow. Extra good.

Golden Swallow-Soft yellow. No markings. Ruffled

Gretchen Zang-Large soft pink shading to scarlet.

Halley-Salmon pink. Very early

Helen Howard-Beautiful golden buff, flushed coral. Outstanding......

Herada-Mauve. Glistening and pure
No. 1

$1 \mathrm{1/2}$ in.

$\$ 3.00$

2.50

1.40

4.00

2.50

2.00

16.00

6.00

4.00

6.00

3.00

2.50

7.00

9.00

2.50

3.00

4.00

3.00

2.20

4.00

10.00

2.00

12.00

30.00

12.50

2.00

2.00

25.00

2.50
No. 2

$1 \frac{1}{4}$ in.

$1 \frac{1 / 2}{}$ in.

$\$ 2.50$

2.25

1.00

3.50

2.25

1.75

13.00

5.00

3.50

5.00

2.50

2.25

6.00

7.75

2.25

2.50

3.50

2.50

1.80

3.50

8.50

1.75

9.00

25.00

10.00

1.75

1.75

20.00

2.10
No. 3

1 to

$1 \frac{1 / 4}{4}$.

$\$ 2.25$

2.00

.80

3.00

2.00

1.50

10.00

4.00

3.00

4.00

2.00

2.00

5.00

6.50

2.00

2.00

3.00

2.25

1.60

3.00

7.00

1.50

6.00

20.00

8.00

1.50

1.50

16.00

1.70 
STANDARD VARIETIES

High Nye-Old rose with yellow throat. Exceedingly tall...

Illuminator-Brilliant carmine red tinted blue. Tall.

Jewell-Light salmon pink, golden throat. Delicate tinting

LeMarshal Foch-Pale pink, large flowers. Early

L'lmmaculee-Elegant pure waxy white. Tall.

Los Angeles-Shrimp pink. Tall.

Maiden's Blush-Pure light pink. Very early

Miss Madison-Clear light pink on white background. Large.

Mona Lisa-Lavender rose, ruffled.

Morning Glory-"The Improved Schweban"

Mrs. Dr. Norton-Delicate cream and pink. Tall and slender.

Mrs. Frances King-Vermillion red. Large and tall.

Mrs. Frank Pendleton-Rose pink, deep red blotch.

Mrs. H. E. Bothin-Flesh pink, scarlet center. Ruffled.

Mrs. Leon Douglas-Begonia rose. Large florets on tall stem.

Mrs. Watt-American Beauty rose.

Myrtle-Delicate pink, nearly white throat. Early

Niagara-Creamy yellow, penciled carmine. Strong grower.

1910 Rose-Rose pink

Opalescent-Light lavender. Fine grower.

Orange Brilliant-Vivid orange. Small flowers. Decorative..

Pink Wonder-Glistening light pink. Extremely large flowers.

Rose Ash-Ashes of roses. Spike tall and very straight.

Richard Deiner-Geranium pink, creamy yellow throat.

Schweban-Canary yellow, red feathering. Large and strong.

Sweet Lavender-Light lavender, dark reddish blotch. Early

Tycko Zang-Salmon pink. Very large flowers.

Virginia-Scarlet, throat darker.

White Wonder-Pure white, flowers large and wide open.

W. H. Pipps-Light rose salmon. Many flowers open.

Yellow Treasure-Clear rich yellow. Ruffled.
No. 1

$1 \frac{1}{2}$ in.

$\$ 7.00$

5.00

4.00

1.40

2.50

3.50

2.00

5.00

4.00

5.00

3.50

1.75

2.50

4.00

6.00

2.50

3.00

2.25

2.00

5.00

2.00

3.00

3.50

4.50

2.00

3.50

3.25

2.20

3.00

15.00

2.00
No. 2

$1^{1 / 4} \mathrm{in}$.

$1 \frac{1}{2} \mathrm{in}$.

$\$ 6.00$

$\$ 5.00$

4.00

3.50

3.25

2.60

1.10

.90

2.25

2.00

3.00

2.50

1.75

1.50

4.25

3.75

3.50

3.00

4.00

3.50

3.00

2.50

1.50

1.20

2.25

2.00

3.50

3.00

5.00

4.25

2.25

2.00

2.50

2.00

2.00

1.80

1.75

1.50

4.25

3.50

1.75

1.50

2.50

2.00

3.00

2.50

4.00

3.50

1.75

1.50

3.00

2.50

2.75

2.50

1.80

1.60

2.50

2.00

13.00

11.00

1.75

1.50 


\section{A FEW OF THE NEWER VARIETIES}

Albatross-New. Pure white, without markings. Wide open....each Buddy Worden-Fine orange tinted pink. Early

Carolus Clusius-Light rose pink. Tall stocky grower.

Gloriana-True salmon with yellow throat. Good propagator. Extraordinary

Helen Phipps-Deeper pink than W. H. Phipps. Earlier and brighter. Many open. Strong grower. Good propagator....100 Helen Wills-Pure white with lemon throat. Very fine............each Lacinatus-Rose pink. Laciniated........................................ doz. Minuet-Wonderful clear lavender. Best of its color.

Mrs. P. W. Sisson-Pure soft cameo pink. Strong grower.

Outstanding variety.

Oriental Silk-Unusual silky textured bloom of deep rose pink

Radiant Orange -Larg Large

Rose Mulberry-Coral-pink flushed violet each

Tobersun-New, pure yellow. Strong grower. Good spike. Many open Strong

Veilchenblau-Dark purplish blue.

Wedgewood-Dark rich violet-purple. Several good size blooms open at one time doz. 100 each doz. each doz. 100 each doz. doz doz. 100

No. 1
$11 / 2$ in.

$\$ 2.25$

.75

5.00

1.25

12.50

15.00

27.00

1.25

1.25

.90

8.50

20.00

1.00

8.00

.75

6.00

1.25

9.00

each doz. each doz

2.00

22.00

.80
No. 2

$1 \frac{1 / 4}{\text { in. }}$

$11 / 2$ in.

$\$ 2.00$

.60

3.50

1.00

10.00

13.00

24.00

1.00

1.10

.75

7.25

17.00

.85

7.25

.60

5.25

1.00

7.50

1.75

18.50

.70

$5.00 \quad 3.50$
No. 3

1 to

$1 \frac{1 / 4}{i n}$.

$\$ 1.75$

2.50

7.50

10.00

20.00

.80

6.25

14.00 .75

5.50

4.25

.80

6.50

1.50

15.00

.60

2.75

\section{VISIT ELM HILL FARM}

Elm Hill Farm is centrally located in one of the great flower growing sections of the United States, being about midway between Cleveland and Pittsburgh on State Route 36 between Akron and Warren, one mile west of Wayland Station and seven miles east of Ravenna, Ohio.

We can supply Planting Stock and Bulblets of many of these varieties. Prices quoted on request. How Many?

We consider shipment by express safer than by mail and will ship that way unless otherwise advised, and postage is included with order.

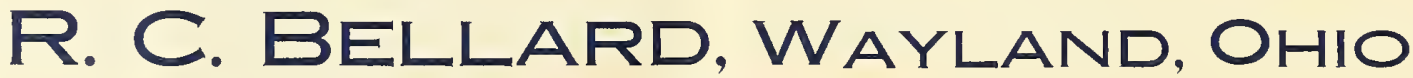

"Home of Elm Hill Gladiolus" 


\section{HELEN PHIPPS BULBLET SPECIAL!}

Having recently acquired quite a large stock of the wonderful pink gladiolus Helen Phipps, we are offering big, plump bulblets for the first time in quantity lots.

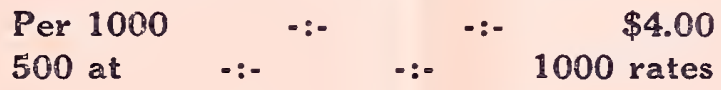

This is a good chance to get a start in this variety. We believe this to be a coming commercial cut flowe: glad. It is a shade deeper, is brighter, and is believed by many to be superior to the well known W. H. Phipps.

Get your order in early, as they won't last long at these prices. 



\section{ORDER SHE T \\ Elm Hill Farm \\ R. C. BELLARD \\ WAYLAND, OHIO \\ WHOLESALE GROWERS OF GLADIOLUS}

Name

WRITE VERY PLAINLY

Street.

City and State

Ship How.

Amount

When.

Enclosed

MAIL OR EXP.

\begin{tabular}{|l|l|l|l|l|l|l|l|}
\hline Quantity & Size & NAME OF VARIETY & \multicolumn{2}{|c|}{ PRICE } & \multicolumn{2}{|c|}{ TOTAL } \\
\hline & & & & & & & \\
\hline & & & & & & \\
\hline & & & & & & & \\
\hline & & & & & & & \\
\hline & & & & & & & \\
\hline & & & & & & & \\
\hline
\end{tabular}


